\title{
Future Directions for Hypothermia following Severe Traumatic Brian Injury
}

\author{
Annie W. Chiu, MS, MPH, MD ${ }^{1}$ Holly E. Hinson, MD, MCR ${ }^{1}$ \\ ${ }^{1}$ Department of Neurocritical Care, Neurology, and Emergency \\ Medicine, Oregon Health \& Science University, Portland, Oregon \\ Semin Respir Crit Care Med 2017;38:768-774.

\begin{abstract}
Address for correspondence Holly E. Hinson, MD, MCR, Department of Neurocritical Care, Neurology, and Emergency Medicine, Oregon Health \& Science University, 3181 SW Sam Jackson Park Road, CR-127,
\end{abstract} \\ Portland, OR 97239 (e-mail: hinson@ohsu.edu).
}

\author{
Abstract \\ Keywords \\ - hypothermia \\ - cooling \\ - fever \\ - traumatic brain injury \\ - intracranial pressure
}

Traumatic brain injury (TBI) is a serious health care problem on both individual and public health levels. As a major cause of death and disability in the United States, it is associated with a significant economic and public health burden. Although the evidence to support the use of induced hypothermia on neurologic outcome after cardiac arrest is well established, its use in treating TBI remains controversial. Hypothermia has the potential to mitigate some of the destructive processes that occur as part of secondary brain injury after TBI. Hypothermia can be helpful in lowering intracranial pressure, for example, but its influence on functional outcome is unclear. There is insufficient evidence to support the broad use of prophylactic hypothermia for neuroprotection after TBI. Investigators are beginning to more carefully select patients for temperature modulating therapies, in a more personalized approach. Examples include targeting immunomodulation and scaling hypothermia to achieve metabolic targets. This review will summarize the clinical evidence for the use of hypothermia to limit secondary brain injury following acute TBI.
Traumatic brain injury (TBI) remains a major cause of death and disability in the United States despite advances in both prevention and treatment. In 2013, approximately 2.8 million emergency department visits, hospital admissions, or deaths were TBI related. ${ }^{1}$ TBI may be classified into three categories guided by initial presenting Glasgow's coma score (GCS). ${ }^{2}$ Mild TBI is defined by a presenting GCS of 13 to 15 , moderate requires a GCS of 8 to 12 , and severe is a GCS of 3 to $8 .^{3}$ While prevention remains key to reducing the public health burden associated with TBI, limiting secondary brain injury after the insult is crucial to improving outcomes.

Reports of hypothermia as treatment for brain injury appear as early as the 1940 s when Fay ${ }^{4}$ cooled a series of severe TBI patients to $28^{\circ} \mathrm{C}$ for up to 7 days. Though the outcomes were better than expected, this and subsequent reports also described deaths related to cooling. For a few decades thereafter, interest in hypothermia had waned. By the 1980s, interest in hypothermia as therapy for TBI was rekindled after experimental studies showed favorable outcomes in animal models of brain injury subjected to moderate hypothermia $\left(32-34^{\circ} \mathrm{C}\right) .^{5}$ Since then, numerous animal studies and clinical trials aimed to better characterize the role of hypothermia in treatment of TBI. This review will summarize the evidence for hypothermia for limiting secondary brain injury following acute TBI in two sections: (1) neuroprotection and (2) rescue therapy.

\section{Rational for Hypothermia: The Biochemical Cascade}

The damage incurred after TBI may be divided into two phases: primary and secondary injuries. The former occurs at the moment of impact, and the latter describes the cascade of damage that ensues after the primary insult. Secondary injury often includes cerebral edema, which may cause increased intracranial pressure (ICP) and decreased cerebral perfusion pressure, leading to ischemia. ${ }^{6}$ The cellular cascade of reactions starts within minutes to hours after injury, and can last days to weeks. Animal studies have shown that
Issue Theme Advancements in Neurocritical Care and Emergency Neurology; Guest Editors: David Y. Hwang, MD, FNCS, and David M. Greer, MD, MA, FCCM, FAHA, FNCS, FAAN, FANA
Copyright (C) 2017 by Thieme Medical Publishers, Inc., 333 Seventh Avenue, New York, NY 10001, USA.

Tel: +1(212) 584-4662.
DOI https://doi.org/ $10.1055 / \mathrm{s}-0037-1607989$. ISSN 1069-3424. 
sustained neuronal hyperexcitability-which is caused by an accumulation of excitatory neurotransmitters, excessive calcium influx into cells, and activation of kinase cascades-can occur within minutes (and last up to days) following injury. Different areas of the brain are physiologically maintained at slightly different temperatures, and such thermodynamic discrepancies may be exacerbated during injury. ${ }^{7}$ Longer acting processes responsible for ongoing secondary brain injury include mitochondrial dysfunction, production of free radicals, blood-brain barrier disruptions, diffusion restriction of cellular membranes, and release of proinflammatory cytokines. Theoretically, the processes producing secondary injury can be modified or even prevented.

In general, hypothermia is thought to be protective against several different aspects of secondary brain injury: reducing hyperexcitability and minimizing thermodynamic disparities, as well as altering the inflammatory response. ${ }^{7}$ Investigators have examined not only the role of hypothermia in interrupting secondary brain injury but also the timing of application of therapeutic hypothermia; both aspects are active areas of investigation.

\section{Hypothermia for Neuroprotection}

The existing literature evaluating prophylactic hypothermia can be generally divided into three categories: (1) hypothermia versus standard of care, (2) timing of hypothermia, and (3) localized versus systemic hypothermia. ${ }^{8}$ As body heat is lost to the environment through four basic mechanismsradiation, convection, evaporation, and conduction-these mechanisms are commonly exploited to achieve systemic hypothermia. These techniques include exposure of skin (radiation), water sprays (evaporation), and fans (convection). Techniques utilizing mechanisms of conduction include water-circulating blankets, ice packs, intravascular catheters, ${ }^{9}$ and noninvasive surface cooling devices with conductive gel pads.

\section{Hypothermia versus Standard of Care}

Inspired by two randomized controlled trials (RCTs) that showed a $15^{10}$ and $18 \%^{11}$ increase in favorable outcome in patients treated with hypothermia (compared with standard management) after severe head injury, a prospective, randomized trial of hypothermia for neuroprotection was conducted. $^{12}$ In this robust multicenter controlled trial (National Acute Brain Injury Study using Hypothermia [NABISH-I]), patients with acute brain injury were randomized to standard care or surface-induced hypothermia (target temperature of $33^{\circ} \mathrm{C}$, maintained for $47 \pm 3$ hours). In the hypothermia group, the target temperature was achieved more than 8 hours after primary injury.

The authors found that hypothermia did not improve the primary outcome measure, the Glasgow's outcome scale (GOS) at 6 months after injury. In fact, the hypothermia group had a higher rate of hypotension and higher percentage of days with complications. Two unexpected benefits of hypothermia were also found: (1) fewer patients in the hypothermia group had elevated ICP and (2) of patients assigned to the hypothermia group, those who were hypothermic on admission had evidence of improved outcomes relative to the group who received standard care. However, this difference was not statistically significant $(p=0.09)$. Within this hypothermic-on-admission patient subgroup, those who were $\leq 45$ years of age showed a significant benefit compared with the standard care group in the same age range $(p=0.02){ }^{12,13}$

Further complicating interpretation of these results, it is known that the induction of hypothermia can lead to episodes of hypovolemia and hypotension ${ }^{14}$-even a single episode of hypotension can significantly worsen clinical outcome in TBI patients, increasing morbidity and possibly doubling mortality. ${ }^{6,14}$

Though the NABISH-I trial was well designed, a common criticism was that the time to target temperature was slow, likely lagging behind the physiologic cascades that were already taking place after initial injury. In addition, the authors later commented that significant intercenter differences in outcomes were present, with more favorable outcomes achieved in larger centers. This was likely due to differences in treatment protocols in the management of hypotension and experience in the use of therapeutic hypothermia. ${ }^{14,15}$ Although many other studies were conducted to evaluate therapeutic hypothermia in $\mathrm{TBI},{ }^{10,11,16-18}$ at that time, the NABISH-I trial was by far the largest (outcome data were obtained for 368 patients) and robustly designed. It heavily influenced the meta-analyses that followed. ${ }^{19,20}$

Despite this high-quality evidence, the role of therapeutic hypothermia in TBI remained controversial. A salient example of this was in 2003 when two meta-analyses published within months of each other, using roughly the same studies, came to divergent conclusions. ${ }^{14,19,20}$

In the Henderson et al meta-analysis, they concluded that there was no clear benefit of induced hypothermia on mortality. However, they found that hypothermia may result in a marginal benefit on neurologic outcome as measured by the GOS. ${ }^{19}$ In this meta-analysis, eight studies were included, resulting in a total of 748 patients. ${ }^{19}$ Given the large number of patients in the NABISH-I trial, it accounted for nearly half of the patients included in the meta-analysis. ${ }^{14}$ In contrast, the McIntyre et al. meta-analysis concluded that therapeutic hypothermia may confer a benefit in mortality and neurological outcomes. In this meta-analysis, 12 studies were included, totaling 1,069 patients. ${ }^{20}$ The NABISH-I patients accounted for approximately $34 \%$ of all patients. ${ }^{14}$

Some authors believe that the Henderson et al analysis included studies with a small number of patients, disparate inclusion criteria, and different treatment protocols. Particularly, problematic was that studies with patients with normal ICPs were combined with studies that required elevated ICP as an inclusion criterion. As variations in ICP may reflect the severity of pathologic progresses that take place after TBI, patients with normal ICP post-TBI may be a distinct category of patients, separate from those with elevated ICP. ${ }^{14}$ Therefore, combining patients with high ICP with the normal ICP group may increase the heterogeneity of 
the subject pool. In addition to the heterogeneous inclusion criteria, differing treatment protocols, and varied sample size between studies, ${ }^{14}$ the time to target temperature was felt to be a likely, if not the essential, factor influencing neurologic outcomes. ${ }^{13-15,21}$

\section{Timing of Hypothermia}

To address the lengthy time to target temperature in their 2001 study, Clifton et al designed the National Acute Brain Injury Study: Hypothermia II (NABIS: H II), a randomized, multicenter clinical trial where the target temperature $\left(33^{\circ}\right.$ C) was achieved in approximately 4.5 hours, cutting the time to target temperature almost in half from the NABISH-I trial. In reducing the time to target temperature, the authors hoped that arresting secondary injury early might improve outcomes. Unfortunately, NABIS: H II was terminated early due to concern about the study's slow recruitment and the feasibility of extending funding without an interim analysis. The study was terminated for futility after an interim analysis that included only 97 patients, despite a planned sample size of 240 patients. ${ }^{22}$

In NABIS: $\mathrm{H}$ II, Clifton et al found no significant difference in poor outcomes between subjects in the hypothermia group compared with the normothermia group; similarly, mortality between the two groups was not significantly different. However, their data suggest that hypothermia may lead to a more complex hospital course: subjects who underwent prophylactic hypothermia had significantly increased rates of raised ICP, received more interventions for raised ICP, and an increase in total rate of complications. ${ }^{22}$ In some aspects, these results were opposite from their 2001 study, in which the hypothermia group had fewer incidents of elevated ICP. ${ }^{12}$ Given the hypothermia-induced hypotension observed in their earlier study, ${ }^{12}$ the authors felt that the most likely explanation for the increased ICP observed in their 2011 study was the aggressive measures taken to reduce such hypotension. ${ }^{22}$

Further, patients with diffuse brain injury who were randomized to the hypothermia group had a greater number of poor outcomes (as defined by GOS scores) and increased mortality compared with their normothermic counterparts ( $p=0.09$ and $p=0.08$, respectively). A possible explanation for these differing outcomes may be that many patients who undergo surgical evacuation may also have bone flaps that were left out, thereby allowing more room for the brain to swell and avoiding increased ICP. Notably, in the surgical intervention group, bone flaps were left out in one-third of the hypothermia patients and nearly half of the normothermia patients. ${ }^{22}$

In both NABISH-I and NABIS: $\mathrm{H}$ II, the target temperature was maintained for only 48 hours, a duration that may be too short to positively affect their primary outcome (the fivecategory GOS score at 6 months). ${ }^{23}$ A Chinese study comparing short-term ( $\sim 2$ days) versus long-term ( $\sim 5$ days) hypothermia found that long-term therapy significantly improved outcome, as measured by the GOS. ${ }^{24}$ They also found that ICP significantly increased in the short-term hypothermia group during rewarming, a change that was unique to the short-term group and not found in the longterm group. ${ }^{24}$ This finding is similar to the increased ICP found in NABIS: H II's hypothermia patients.

In animal models, ischemia has been found to occur in the context of hematoma expansion, its removal, and subsequent reperfusion. ${ }^{25}$ As intraischemic hypothermia has been shown to improve secondary injury in animal models, ${ }^{26}$ it is possible that a similar effect was seen in vivo in this study. Given that diffuse brain injury is not associated with ischemia, the authors felt that this was a possible explanation for the discrepant outcomes in the subgroup analysis. ${ }^{22}$

The current Brain Trauma Foundation (BTF) guidelines recommend against early ( $\leq 2.5$ hours) and short-term (48 hours postinjury) prophylactic hypothermia due to insufficient evidence. ${ }^{8}$ Presently, the Prophylactic Hypothermia Trial to Lessen Traumatic Brain Injury (POLAR) study is underway with the goal to evaluate prophylactic moderate hypothermia $\left(33^{\circ} \mathrm{C}\right)$ initiated within 3 hours of injury and continued for at least 72 hours. Recruitment began in December 2010 and is expected to end in December $2017 .^{27}$

\section{Selective Brain Cooling versus Systemic Hypothermia}

To mitigate the risk associated with systemic hypothermia (e.g., shock, infection, coagulopathy), subsequent rewarming (e.g., infection, shock), and to achieve a faster target temperature, some groups have investigated selective brain cooling as an alternate means of achieving the goals of hypothermia. Multiple methods for selective cerebral hypothermia have been proposed, including surface cooling, intranasal cooling, transvenous endovascular cooling, ${ }^{28}$ and brain surface irrigation. ${ }^{29}$

Although several studies have examined selective cooling, ${ }^{29,30}$ the highest quality data to date comes from a 2006 investigation in China. Researchers conducted a randomized, controlled, double-blinded study comparing selective brain cooling against systemic cooling and normothermia (control, $37^{\circ} \mathrm{C}$ ) in patients with severe TBI (history of TBI, GCS $\leq 8$ on admission, and brain injury evident on CT scan within 24 hours of admission). To selectively cool the brain to 33 to $35^{\circ} \mathrm{C}$, patients wore a cooling cap with circulating $4^{\circ} \mathrm{C}$ circulating water and a neckband with ice strips. Target temperature in systemic hypothermia $\left(33-35^{\circ} \mathrm{C}\right)$ group was achieved through use of a cooling blanket and ice bags..$^{31}$

Researchers found that GOS were highest (GOS score of 4 or 5 ) in the selective brain cooling group 2 years after injury compared with systemic hypothermia and the control groups $(72.7 \%$ in selective brain cooling group, $57.1 \%$ in systemic hypothermia group, and $34.8 \%$ in control group). In addition, the frequency of good neurologic outcome (defined as GOS score of 4 or 5 ) was significantly higher in the hypothermia groups compared with the normothermia group. The selective brain cooling group also had significantly lower ICPs relative to the control group. This result should be interpreted with caution, however, as the average ICP value in all groups, at all time points, was above $20 \mathrm{~mm} \mathrm{Hg}^{31}$ 
Systemic complications appeared to be mitigated with focal cooling-the group found that rates of pneumonia were lowest in the selective brain cooling group. Though both hypothermia groups had evidence of thrombocytopenia, platelet counts returned to normal within 3 days of rewarming. ${ }^{31}$ Finally, the authors observed that superoxide dismutase levels were significantly higher on days 3 and 7 postinjury in the hypothermia groups compared with the normothermia groups, which might reflect hypothermia's ability to reduce free radical generation and lipid peroxidation. $^{31}$ Though promising, the evidence at present is insufficient to formulate guidelines regarding selective versus systemic hypothermia in TBI. ${ }^{8}$

\section{Hypothermia for Rescue Therapy}

\section{Elevated Intracranial Pressure}

ICP measurements exceeding $20 \mathrm{~mm} \mathrm{Hg}$ are regarded as intracranial hypertension, ${ }^{32}$ though emerging evidence suggests this threshold might not apply to all patients. ${ }^{33}$ After TBI, ICP can rise due to hematoma expansion, cerebral edema, or impaired autoregulation. ${ }^{34}$ Retrospective studies from large databanks have shown an association between elevated ICP ( $\geq 20 \mathrm{~mm} \mathrm{Hg}$ ) initially or during clinical deterioration and both neurologic worsening and poor outcome. $^{35}$ Though elevated ICP is associated with worse outcome, it remains unclear whether increased ICP is reflective of ongoing pathological processes after TBI, an independent factor complicating a patient's clinical course, or both. ${ }^{14,36}$ In the most recent iteration of the BTF guidelines, treatment of ICP $>22 \mathrm{~mm} \mathrm{Hg}$ is recommended as level II B recommendation. Values exceeding $22 \mathrm{~mm} \mathrm{Hg}$ are associated with increased mortality. ${ }^{8}$ Historically, high ICP has been treated with a tiered approach, moving from less invasive to more invasive measures (e.g., head elevation, osmotic therapy, and surgical intervention). ${ }^{32}$

Hypothermia is a tool in the armamentarium for both cerebral edema and elevated ICP. Numerous studies have demonstrated hypothermia to be effective in controlling elevated ICP, and can be used to control intracranial hypertension even in the later stages after initial injury. A metaanalysis of eight RCTs examining therapeutic hypothermia for severe TBI found that hypothermia can lower ICP even in patients refractory to first-line therapies. ${ }^{19}$ However, it remained unclear whether functional outcome is improved by use of hypothermia in this context. ${ }^{7}$

To address the question of functional outcome, European investigators conducted the Eurotherm3235 trial, a large, multicenter RCT where patients with severe TBI and refractory raised ICP ( $>20 \mathrm{~mm} \mathrm{Hg}$ for $>5$ minutes) were randomized to receive second-tier interventions or second-tier interventions with therapeutic hypothermia $\left(32-35^{\circ} \mathrm{C}\right)$. In contrast to common practice in the United States, secondtier interventions included osmotherapy and inotropes. In the hypothermia group, the core temperature was reduced to the highest temperature necessary to maintain an ICP of $\leq 20 \mathrm{~mm} \mathrm{Hg}$. Hypothermia was maintained for at least 48 hours and continued for as long as necessary to keep
ICP within goal. For patients in both groups who could not achieve ICP $\leq 20 \mathrm{~mm} \mathrm{Hg}$, stage 3 interventions were pursued. Stage 3 interventions included barbiturate therapy and decompressive craniectomy. The primary outcome was the GOS-extended score at 6 months. ${ }^{37}$

Compared with controls, fewer hypothermia patients required escalation of care to control ICP. However, the trial was terminated early due to safety concerns: the distribution of GOS-extended scores and number of adverse events reflected a worse outcome in the hypothermia group. ${ }^{37}$ Although the Eurotherm3235 data support hypothermia's role in effectively controlling ICP, it further fuels the debate as to whether reducing ICP translates into improved neurological outcomes. ${ }^{36,38,39}$

\section{Immunomodulation: Hypothermia's Effect on Cytokines or Inflammatory Markers}

As a result of the initial injury, a robust inflammatory cascade occurs both centrally and peripherally. This cascade is characterized by a spectrum of increased cytokine production, as demonstrated in both animal models ${ }^{40-42}$ and clinical studies. ${ }^{43-53}$ Analysis of post-TBI brain tissue in animal models reveals robust local inflammation about the area of injury. ${ }^{40-42}$ In humans, elevated levels of proinflammatory cytokines such as interleukin (IL)-1, ${ }^{43,44}$ IL-6, ${ }^{43,45-49}$ and tumor necrosis factor- $\alpha^{53}$ in cerebrospinal fluid suggest intrathecal production, as well as peripheral expression measured in blood. The initial expression of proinflammatory cytokines (systemic inflammatory response) is followed by a second, delayed phase of restorative, immunosuppressive release of cytokines including IL-10 (compensatory antiinflammatory response) that may be associated with immunosuppression. ${ }^{54,55}$

Experimentally, hypothermia after moderate TBI (using a fluid percussion injury model) was associated with significantly higher levels of growth-associated protein (GAP-43) in brain parenchyma. ${ }^{56}$ GAP-43 is thought to be a marker of neuronal sprouting, leading the authors to conclude that hypothermia may encourage neuronal sprouting post-TBI. The examiners also found lower levels of IL-6 and cytokine signaling 3 (SOCS3) in the hypothermia group. Suppressor of SOCS3 is believed to be a key negative regulator, ${ }^{57}$ and its expression is believed to inhibit cell survival and outgrowth after injury. ${ }^{58}$ This is in accordance with previous data which showed that post-TBI hypothermia can attenuate plasma IL-6 levels in humans. ${ }^{59}$ Given the complexity of the cellular cascades, further investigation is needed to clarify hypothermia's role post-TBI.

\section{Metabolic-Targeted Hypothermia}

In contrast to targeting a specific temperature for all patients, Feng et al offered a more personalized approach to therapeutic hypothermia in a pilot RCT. As hypothermia is thought to reduce the metabolic rate, the investigators tailored the cooling to achieve a target metabolic rate rather than a strict temperature goal. 
In this single-blind RCT, severe TBI patients (defined as GCS 3-8) were assigned to either the metabolic-targeted hypothermia treatment (MTHT) group or the body temperature-targeted hypothermia treatment (BTHT) control group. In the MTHT group, hypothermia was employed to achieve 50 to $60 \%$ of a patient's rest metabolic rate for 5 days. (Each patient's resting metabolic rate was measured by an indirect calorimetry management system.) In the set temperature group, 32 to $35^{\circ} \mathrm{C}$ was set as the target temperature and maintained for 5 days. The primary outcome of the study was mortality. Nested within this study was a metabolomics analysis where blood samples were analyzed through use of a proton nuclear magnetic resonance. This was done to explore changes in metabolic patterns in both the hypothermia and control groups.

In the pilot phase, the investigators found that the mortality rate was lower in the MTHT group compared with the temperature-targeted hypothermia treatment group $(p=0.049)$. Though the sample size was relatively small (88 patients), the results are encouraging. Of note, of the 42 MTHT cases who completed therapy, 4 cases withdrew, 2 cases abandoned treatment, and 2 cases died. The investigators are actively recruiting patients for a more definitive study. ${ }^{60}$

\section{Fever/Targeted Temperature Management}

Some investigators have posited that the benefits of hypothermia are related to strict fever avoidance. Fever is common in severe TBI and may contribute to secondary brain injury. ${ }^{61}$ Even small fluctuations in systemic and local (brain) temperature can affect neuronal vulnerability after injury. ${ }^{62}$ To assess the effect of fever on patient outcome and mortality, Li and Jiang (2012) conducted a retrospective analysis of data from the Chinese Head Trauma Data Bank. Patients were divided into three groups: normothermia $\left(36.3-37.2^{\circ} \mathrm{C}\right)$ and mild fever $\left(37.3-38.0^{\circ} \mathrm{C}\right)$, moderate fever $\left(38.1-39.0^{\circ} \mathrm{C}\right)$, and high fever $\left(>39.0^{\circ} \mathrm{C}\right)$. Statistically significant differences in the mortality rate and unfavorable outcomes (defined as severe disability, vegetative state, and death as measured on the GOS [1-3]) were found between the groups: Both mortality rates and unfavorable outcomes increased with the degree of pyrexia. ${ }^{63}$ Their results suggest that posttraumatic hyperthermia exerts a deleterious effect that is proportional to both its degree and duration. The mechanism of its effect likely involves the many and complex processes involved in secondary injury.

Strict fever avoidance contrasts to standard management in that standard care only addresses elevated temperatures after they occur, usually with antipyretic medication. In contrast, targeted temperature management (TTM) is a process that achieves a specific temperature, often with the intent to tightly control temperature to mitigate potentially harmful processes that occur during secondary injury.

In 2016, Miyata et al compared the effect of therapeutic hypothermia $\left(32-34^{\circ} \mathrm{C}\right)$ and TTM $\left(35.5-37^{\circ} \mathrm{C}\right)$ on GOS scores at 6 months in patients with severe TBI. They found no significant difference in the likelihood of poor neurologic outcome and mortality between patients treated with hypothermia and those treated with fever control. In addition, they found that the rate of complications (secondary outcome) was significantly higher in the hypothermia group compared with the fever control group, suggesting that hypothermia is not only noninferior to fever control but that hypothermia may cause harm. Although the results of this study are compelling, its conclusions must be interpreted with caution: though the goal was to achieve target temperature within 6 hours of injury, over half (65\%) of the patients in the hypothermia group took over 6 hours to reach their target temperature.

A recent cohort observational study from the Nationwide Japan Neurotrauma Data Bank offers further insight. In this observational study, investigators divided TBI patients into two groups: those who underwent therapeutic temperature management of any kind versus conventional management. The former group included both therapeutic normothermia $\left(<37^{\circ} \mathrm{C}\right)$ and therapeutic hypothermia $\left(<35^{\circ} \mathrm{C}\right)$ patients. The control group was not exposed to mechanical cooling devices but did receive medical antipyretic drugs if medically necessary. No significant difference in GOS was found between the two groups at discharge. ${ }^{64}$

\section{Conclusion}

Though there is compelling rationale that hypothermia has the potential to mitigate the destructive metabolic processes that occur during secondary injury, there has yet to be strong and clear evidence to support its broad use in severe TBI to modify long-term outcomes. Hypothermia does seem to be a reasonable option to control elevated ICP in select patients. The future of hypothermia and TTM likely lay in careful patient selection and customization to achieve specific clinical targets. This approach may take the form of exploring hypothermia's role in post-TBI immunomodulation and achieving metabolic targets, in a more personalized approach to therapy.

\section{References}

1 Taylor CA, Bell JM, Breiding MJ, Xu L. Traumatic brain injuryrelated emergency department visits, hospitalizations, and deaths - United States, 2007 and 2013. MMWR Surveill Summ 2017;66(09):1-16

2 Teasdale G, Jennett B. Assessment of coma and impaired consciousness. A practical scale. Lancet 1974;2(7872):81-84

3 Miller JD. Minor, moderate and severe head injury. Neurosurg Rev 1986;9(1-2):135-139

4 Fay T. Observations on generalized refrigeration in cases of severe cerebral trauma. Assoc Res Nerv Ment Dis 1943;24:611-619

5 Bernard SA, Buist M; MacC Jones B. Experience with prolonged induced hypothermia in severe head injury. Crit Care 1999;3(06): 167-172

6 Ghajar J. Traumatic brain injury. Lancet 2000;356(9233):923-929

7 Polderman KH. Induced hypothermia and fever control for prevention and treatment of neurological injuries. Lancet 2008;371 (9628):1955-1969

8 Carney N, Totten AM, O'Reilly C, et al. Guidelines for the management of severe traumatic brain injury, fourth edition. Neurosurgery $2017 ; 80(01): 6-15$ 
9 Polderman KH. Application of therapeutic hypothermia in the intensive care unit. Opportunities and pitfalls of a promising treatment modality-part 2: practical aspects and side effects. Intensive Care Med 2004;30(05):757-769

10 Clifton GL, Allen S, Barrodale P, et al. A phase II study of moderate hypothermia in severe brain injury. J Neurotrauma 1993;10(03): 263-271, discussion 273

11 Marion DW, Obrist WD, Carlier PM, Penrod LE, Darby JM. The use of moderate therapeutic hypothermia for patients with severe head injuries: a preliminary report. J Neurosurg 1993;79(03): 354-362

12 Clifton GL, Miller ER, Choi SC, et al. Lack of effect of induction of hypothermia after acute brain injury. N Engl J Med 2001;344(08): 556-563

13 Clifton GL, Miller ER, Choi SC, et al. Hypothermia on admission in patients with severe brain injury. J Neurotrauma 2002;19(03): 293-301

14 Polderman KH, Ely EW, Badr AE, Girbes ARJ. Induced hypothermia in traumatic brain injury: considering the conflicting results of meta-analyses and moving forward. Intensive Care Med 2004;30 (10): $1860-1864$

15 Clifton GL, Choi SC, Miller ER, et al. Intercenter variance in clinical trials of head trauma-experience of the National Acute Brain Injury Study: hypothermia. J Neurosurg 2001;95(05):751-755

16 Shiozaki T, Hayakata T, Taneda M, et al; Mild Hypothermia Study Group in Japan. A multicenter prospective randomized controlled trial of the efficacy of mild hypothermia for severely head injured patients with low intracranial pressure. J Neurosurg 2001;94(01):50-54

17 Jiang J, Yu M, Zhu C. Effect of long-term mild hypothermia therapy in patients with severe traumatic brain injury: 1-year follow-up review of 87 cases. J Neurosurg 2000;93(04):546-549

18 Qiu WS, Liu WG, Shen H, et al. Therapeutic effect of mild hypothermia on severe traumatic head injury. Chin J Traumatol 2005;8(01):27-32

19 Henderson WR, Dhingra VK, Chittock DR, Fenwick JC, Ronco JJ. Hypothermia in the management of traumatic brain injury. A systematic review and meta-analysis. Intensive Care Med 2003; 29(10):1637-1644

20 McIntyre LA, Fergusson DA, Hébert PC, Moher D, Hutchison JS. Prolonged therapeutic hypothermia after traumatic brain injury in adults: a systematic review. JAMA 2003;289(22):2992-2999

21 Polderman KH. Application of therapeutic hypothermia in the ICU: opportunities and pitfalls of a promising treatment modality. Part 1: indications and evidence. Intensive Care Med 2004;30 (04):556-575

22 Clifton GL, Valadka A, Zygun D, et al. Very early hypothermia induction in patients with severe brain injury (the National Acute Brain Injury Study: Hypothermia II): a randomised trial. Lancet Neurol 2011;10(02):131-139

23 Maas A, Stocchetti N. Hypothermia and the complexity of trials in patients with traumatic brain injury. Lancet Neurol 2011;10(02): $111-113$

24 Jiang J-Y, Xu W, Li W-P, et al. Effect of long-term mild hypothermia or short-term mild hypothermia on outcome of patients with severe traumatic brain injury. J Cereb Blood Flow Metab 2006;26 (06):771-776

25 Kuroda Y, Bullock R. Local cerebral blood flow mapping before and after removal of acute subdural hematoma in the rat. Neurosurgery 1992;30(05):687-691

26 Burger R, Bendszus M, Vince GH, Solymosi L, Roosen K. Neurophysiological monitoring, magnetic resonance imaging, and histological assays confirm the beneficial effects of moderate hypothermia after epidural focal mass lesion development in rodents. Neurosurgery 2004;54(03):701-711, discussion 711-712

27 Nichol A, Gantner D, Presneill J, et al. Protocol for a multicentre randomised controlled trial of early and sustained prophylactic hypothermia in the management of traumatic brain injury. Crit Care Resusc 2015;17(02):92-100
28 Christian E, Zada G, Sung G, Giannotta SL. A review of selective hypothermia in the management of traumatic brain injury. Neurosurg Focus 2008;25(04):E9

29 Idris Z, Zenian MS, Muzaimi M, Hamid WZWA. Better Glasgow outcome score, cerebral perfusion pressure and focal brain oxygenation in severely traumatized brain following direct regional brain hypothermia therapy: a prospective randomized study. Asian J Neurosurg 2014;9(03):115-123

30 Qiu W, Shen H, Zhang Y, et al. Noninvasive selective brain cooling by head and neck cooling is protective in severe traumatic brain injury. J Clin Neurosci 2006;13(10):995-1000

31 Liu WG, Qiu WS, Zhang Y, Wang WM, Lu F, Yang XF. Effects of selective brain cooling in patients with severe traumatic brain injury: a preliminary study. J Int Med Res 2006;34 (01):58-64

32 Sahuquillo J, Arikan F. Decompressive craniectomy for the treatment of refractory high intracranial pressure in traumatic brain injury. Cochrane Database Syst Rev 2006;(01):CD003983

33 Chesnut RM, Temkin N, Carney N, et al; Global Neurotrauma Research Group. A trial of intracranial-pressure monitoring in traumatic brain injury. N Engl J Med 2012;367(26):2471-2481

34 Stocchetti N, Maas AIR. Traumatic intracranial hypertension. N Engl J Med 2014;370(22):2121-2130

35 Juul N, Morris GF, Marshall SB, Marshall LF; The Executive Committee of the International Selfotel Trial. Intracranial hypertension and cerebral perfusion pressure: influence on neurological deterioration and outcome in severe head injury. J Neurosurg 2000;92(01):1-6

36 Lazaridis C, Robertson CS. Hypothermia for increased intracranial pressure: is it dead? Curr Neurol Neurosci Rep 2016;16 (09):78

37 Andrews PJ, Sinclair HL, Rodriguez A, et al; Eurotherm3235 Trial Collaborators. Hypothermia for intracranial hypertension after traumatic brain injury. N Engl J Med 2015;373(25):2403-2412

38 Robertson CS, Ropper AH. Getting warmer on critical care for head injury. N Engl J Med 2015;373(25):2469-2470

39 Cooper DJ, Rosenfeld JV, Murray L, et al; DECRA Trial Investigators; Australian and New Zealand Intensive Care Society Clinical Trials Group. Decompressive craniectomy in diffuse traumatic brain injury. N Engl J Med 2011;364(16):1493-1502

40 Taupin V, Toulmond S, Serrano A, Benavides J, Zavala F. Increase in IL-6, IL-1 and TNF levels in rat brain following traumatic lesion. Influence of pre- and post-traumatic treatment with Ro5 4864, a peripheral-type ( $p$ site) benzodiazepine ligand. J Neuroimmunol 1993;42(02):177-185

41 Shohami E, Novikov M, Bass R, Yamin A, Gallily R. Closed head injury triggers early production of TNF alpha and IL- 6 by brain tissue. J Cereb Blood Flow Metab 1994;14(04):615-619

42 Kita T, Liu L, Tanaka N, Kinoshita Y. The expression of tumor necrosis factor-alpha in the rat brain after fluid percussive injury. Int J Legal Med 1997;110(06):305-311

43 Singhal A, Baker AJ, Hare GMT, Reinders FX, Schlichter LC, Moulton RJ. Association between cerebrospinal fluid interleukin- 6 concentrations and outcome after severe human traumatic brain injury. J Neurotrauma 2002;19(08):929-937

44 McClain CJ, Cohen D, Ott L, Dinarello CA, Young B. Ventricular fluid interleukin-1 activity in patients with head injury. J Lab Clin Med 1987;110(01):48-54

45 Bell MJ, Kochanek PM, Doughty LA, et al. Interleukin-6 and interleukin-10 in cerebrospinal fluid after severe traumatic brain injury in children. J Neurotrauma 1997;14(07):451-457

46 McClain C, Cohen D, Phillips R, Ott L, Young B. Increased plasma and ventricular fluid interleukin-6 levels in patients with head injury. J Lab Clin Med 1991;118(03):225-231

47 Kossmann T, Hans V, Imhof HG, Trentz O, Morganti-Kossmann MC. Interleukin-6 released in human cerebrospinal fluid following traumatic brain injury may trigger nerve growth factor production in astrocytes. Brain Res 1996;713(1-2):143-152 
48 Hans VH, Kossmann T, Joller H, Otto V, Morganti-Kossmann MC. Interleukin-6 and its soluble receptor in serum and cerebrospinal fluid after cerebral trauma. Neuroreport 1999;10(02):409-412

49 Nwachuku EL, Puccio AM, Adeboye A, Chang Y-F, Kim J, Okonkwo DO. Time course of cerebrospinal fluid inflammatory biomarkers and relationship to 6-month neurologic outcome in adult severe traumatic brain injury. Clin Neurol Neurosurg 2016;149:1-5

50 Kossmann T, Stahel PF, Lenzlinger PM, et al. Interleukin-8 released into the cerebrospinal fluid after brain injury is associated with blood-brain barrier dysfunction and nerve growth factor production. J Cereb Blood Flow Metab 1997;17(03):280-289

51 Whalen MJ, Carlos TM, Kochanek PM, et al. Interleukin-8 is increased in cerebrospinal fluid of children with severe head injury. Crit Care Med 2000;28(04):929-934

52 Stahel PF, Kossmann T, Joller H, Trentz O, Morganti-Kossmann MC. Increased interleukin-12 levels in human cerebrospinal fluid following severe head trauma. Neurosci Lett 1998;249(2-3):123-126

53 Ross SA, Halliday MI, Campbell GC, Byrnes DP, Rowlands BJ. The presence of tumour necrosis factor in CSF and plasma after severe head injury. Br J Neurosurg 1994;8(04):419-425

54 Knoblach SM, Faden AI. Interleukin-10 improves outcome and alters proinflammatory cytokine expression after experimental traumatic brain injury. Exp Neurol 1998;153(01):143-151

55 Kremlev SG, Palmer C. Interleukin-10 inhibits endotoxin-induced pro-inflammatory cytokines in microglial cell cultures. J Neuroimmunol 2005;162(1-2):71-80

56 Zhao C-C, Wang C-F, Li W-P, et al. Mild hypothermia promotes pericontusion neuronal sprouting via suppressing suppressor of cytokine signaling 3 expression after moderate traumatic brain injury. J Neurotrauma 2017;34(08):1636-1644
57 Liu X, Williams PR, He Z. SOCS3: a common target for neuronal protection and axon regeneration after spinal cord injury. Exp Neurol 2015;263:364-367

58 Park KW, Lin C-Y, Lee Y-S. Expression of suppressor of cytokine signaling-3 (SOCS3) and its role in neuronal death after complete spinal cord injury. Exp Neurol 2014;261:65-75

59 Aibiki M, Maekawa S, Ogura S, Kinoshita Y, Kawai N, Yokono S. Effect of moderate hypothermia on systemic and internal jugular plasma IL-6 levels after traumatic brain injury in humans. J Neurotrauma 1999;16(03):225-232

60 Feng J-Z, Wang W-Y, Zeng J, et al. Optimization of brain metabolism using metabolic-targeted therapeutic hypothermia can reduce mortality from traumatic brain injury. J Trauma Acute Care Surg 2017;83(02):296-304

61 Rincon F, Hunter K, Schorr C, Dellinger RP, Zanotti-Cavazzoni S. The epidemiology of spontaneous fever and hypothermia on admission of brain injury patients to intensive care units: a multicenter cohort study. J Neurosurg 2014;121(04):950-960

62 Dietrich WD, Bramlett HM. Therapeutic hypothermia and targeted temperature management in traumatic brain injury: clinical challenges for successful translation. Brain Res 2016;1640(Pt A):94-103

63 Li J, Jiang JY. Chinese Head Trauma Data Bank: effect of hyperthermia on the outcome of acute head trauma patients. J Neurotrauma 2012;29(01):96-100

64 Miyata K, Ohnishi H, Maekawa K, et al. Therapeutic temperature modulation in severe or moderate traumatic brain injury: a propensity score analysis of data from the Nationwide Japan Neurotrauma Data Bank. J Neurosurg 2016;124(02): 527-537 